\title{
PARASITOSES INTESTINAIS EM ESTUDANTES DE UMA ESCOLA PÚBLICA DO MUNICÍPIO DE SÃO MATEUS, ESPÍRITO SANTO, BRASIL
}

\author{
INTESTINAL PARASITOSES IN STUDENTS IN A PUBLIC SCHOOL IN THE \\ MUNICIPALITY OF SÃO MATEUS, ESPÍRITO SANTO, BRAZIL
}

\author{
Heitor Pivetta ${ }^{1}$, Janaina dos Santos Martins ${ }^{2}$, \& Marco Antônio Andrade de Souza ${ }^{3 *}$ \\ 123 Departamento de Ciências da Saúde do Centro Universitário Norte do Espírito Santo da Universidade \\ Federal do Espírito Santo. ${ }^{1}$ heitor.pivetta@gmail.com ${ }^{2}$ janaina-santos45@live.com ${ }^{3 *}$ marco.souza@ufes.br
}

\section{ARTIGO INFO.}

Recebido em: 03.01.2021

Aprovado em: 25.01.2021

Disponibilizado em: 05.02.2021

\section{Palavras-chave:}

Parasitologia; Prevalência; Educação; População Infantil; Questionário.

\section{KEYWORDS:}

Parasitology; Prevalence, Education; Child Population; Questionnaire.

\section{*Autor Correspondente: Souza, M. A. A. de. \\ RESUMO}

O caráter endêmico em algumas regiões do Brasil para doenças causadas por enteroparasitos ainda é bastante evidenciado e poucas ações têm sido apresentadas pelos órgãos governamentais para mudar esse quadro. Considerando que a população infantil é a mais acometida por doenças parasitológicas, o presente estudo analisou amostras de fezes de estudantes de uma escola pública do município de São Mateus, Espírito Santo, Brasil e desenvolveu atividades educativas e de conscientização junto à população em estudo. A pesquisa foi de natureza descritiva, privilegiando uma abordagem interpretativa. Amostras de fezes dos participantes foram coletadas no período de abril a junho de 2019, após assinatura de um termo de consentimento livre e esclarecido pelos pais ou responsáveis. O material foi encaminhando ao Laboratório de Parasitologia e Hematologia Clínica do Centro Universitário Norte do Espírito Santo, da Universidade Federal do Espírito Santo e analisado pelo método de sedimentação espontânea. De um total de 52 frascos coletores distribuídos na escola, entre os indivíduos que concordaram em participar da pesquisa, 22 amostras $(42,31 \%)$ foram recolhidas. Destas, $8(36,36 \%)$ estavam positivas para cisto de protozoários e ovos de helmintos. O comensal Entamoeba coli $(12,5 \%)$ e os parasitos Entamoeba histolytica/dispar $(62,5 \%)$ e Trichuris trichiura
(25\%) foram os mais prevalentes. Os resultados permitem compreender a relevância necessidade da aplicação de medidas de educação sanitária e de investimentos no serviço público, a fim de minimizar os processos de transmissão parasitária, principalmente em regiões que apresentam precariedades sociais.

\begin{abstract}
The endemic character in some regions of Brazil for diseases caused by enteroparasites is still quite evident and few actions have been presented by government agencies to change this situation. Considering that the child population is the most affected by parasitological diseases, the present study analyzed stool samples from students in a public school in the city of São Mateus, Espírito Santo, Brazil and developed educational and awareness activities with the study population. The research was a descriptive nature, favoring an interpretative approach. Stool samples from participants were collected from April to June 2019, after signing a free and informed consent form by parents or guardians. The material was sent to Laboratório de Parasitologia e Hematologia Clínica of the Centro Universitário Norte do Espírito Santo, at the Universidade Federal do Espírito Santo and analyzed by the spontaneous sedimentation method. From a total of 52 collection vials distributed at the school among the individuals, who agreed to participate in the research, 22 samples (42.31\%) were collected. Of these, $8(36.36 \%)$ were positive for protozoan cyst and helminth eggs. Entamoeba coli (12.5\%) commensal and Entamoeba histolytica/dispar $(62.5 \%)$ and Trichuris trichiura (25\%) parasites were the most prevalent. The results allow us to understand the relevance of the need for the application of health education measures and investments in the public service, in order to minimize the processes of parasitic transmission, especially in regions with social precariousness.
\end{abstract}




\section{INTRODUÇÃO}

As parasitoses intestinais constituem um importante problema de saúde pública, principalmente pelos efeitos que podem ocasionar sobre os estados físico, nutricional e mental dos indivíduos (Cimerman, \& Cimerman, 2001). Acometem um elevado percentual da população humana, especialmente os indivíduos que vivem em comunidades de baixa renda, afetando o desenvolvimento das crianças e limitando suas atividades em geral (Tavares et al., 2001).

A maioria das infecções causadas pelos parasitos costuma ser adquirida por via oral, através da contaminação fecal da água e alimentos e são mais endêmicas em países com más condições de saneamento, que exigem consideráveis recursos financeiros, organização e pessoal habilitado para combatê-las (Tavares et al., 2001).

As infecções parasitárias são provocadas por helmintos e protozoários que colonizam o intestino dos indivíduos. Apresentam ampla distribuição geográfica, ocorrendo em áreas urbanas e rurais, com variações de acordo o ambiente e espécie de parasito envolvido (Santos et al., 2017).

Um protozoário constitui-se de uma única célula que, para sobreviver, realiza todas as funções mantenedoras da vida: alimentação, respiração, reprodução, excreção e locomoção. O grupo dos protozoários é constituído por mais de 60.000 espécies conhecidas, das quais $50 \%$ são fósseis e o restante vive até hoje. Destes, aproximadamente 10.000 espécies são parasitos dos mais variados animais e apenas algumas dezenas de espécies infectam o homem (Neves et al., 2016).

Quanto a morfologia, apresentam grande variações, conforme sua fase evolutiva e meio que estejam adaptados. Podem ser esféricos, ovais ou mesmo alongados. Alguns são revestidos de cílios, outros possuem flagelos e existem, ainda, os que não possuem nenhuma organela locomotora especializada. Dependendo da sua atividade fisiológica, algumas espécies possuem fases bem definidas (Neves et al., 2016).

Além dos protozoários destacam-se os helmintos, que constituem um grupo muito numeroso de animais, incluindo espécies de vida livre e de vida parasitária, que se apresentam distribuídos nos filos Platyhelminthes, Nematoda e Acanthocephala. Alguns Gordiáceos, representantes do filo Nematomorpha e conhecidos como "crina de cavalo", parasitos essencialmente de invertebrados, podem ocasionalmente causar parasitismo passageiro no ser humano sem graves consequências (Ferraz et al., 2014; Neves et al., 2016).

As ocorrências de helmintos no homem são muito comuns. De fato, cerca de $20 \%$ da população humana no mundo estão parasitados por ancilostomídeos, o que equivale a mais de um bilhão de pessoas. A situação é equivalente em relação ao Ascaris lumbricoides. Estas infecções, em geral, resultam para o hospedeiro danos que se manifestam de formas variadas (Neves et al., 2016). 
A Organização Mundial de Saúde alerta sobre a alta frequência das doenças parasitárias na população mundial, estimando que cerca de 980 milhões de pessoas estejam parasitadas pelo A. lumbricoides e 200 milhões pelo Schistosoma mansoni (Lodo et al., 2010).

No Brasil, a prevalência de enteroparasitoses é sabidamente elevada, principalmente na população pediátrica (0-5 anos) e em crianças em idade escolar e as peculiaridades ambientais como clima quente, umidade, temperatura, presença abundante de vetores e hospedeiros intermediários contribuem, significativamente, para o desenvolvimento dos parasitos (Damázio, Soares, \& Souza, 2016; Neves et al., 2016; Ludwig, \& Conte, 2017).

O índice das parasitoses intestinais, embora no Brasil tenha decrescido nos últimos anos, ainda é considerado elevado, razão pela qual o exame parasitológico de fezes (EPF) deveria assumir maior importância na clínica médica. Muitas vezes o EPF não faz parte dos exames complementares solicitados, pelo fato de as parasitoses intestinais não estarem incluídas na hipótese diagnosticada e pelo fato de o clínico negligenciá-lo. Este é um exame barato, não invasivo, de fácil execução, que, quando bem executado, pode evitar a realização de exames mais invasivos, como endoscopia digestiva, biopsias ou exame de imagem (Neves et al., 2016).

Braz et al. (2015, p. 369) afirmam que "os parasitas mais comuns atualmente em crianças são A. lumbricoides, Ancylostoma duodenale e o Trichuris trichiura, mas também podem ser encontrados Giardia lamblia e Entamoeba histolytica." Os danos que esses parasitos podem causar a seus portadores incluem, entre outros agravos, a obstrução intestinal ( $A$. lumbricoides), a desnutrição (A. lumbricoides e T. trichiura), a anemia por deficiência de ferro (ancilostomídeos) e quadros de diarreia e de má absorção (E. histolytica e G. lamblia), sendo que as manifestações clínicas são usualmente proporcionais à carga parasitária albergada pelo indivíduo (Ludwig, \& Conte, 2017).

No Espírito Santo, segundo dados do Sistema Único de Saúde, há centenas de casos de parasitoses em jovens capixabas, especialmente na faixa etária de 7 e 17 anos (BRASIL, 2011; 2018). Por outro lado, em São Mateus, norte do Estado do Espírito Santo, ainda há uma enorme carência de estudos sistemáticos que forneçam um dimensionamento epidemiológico das enteroparasitoses, sobretudo em crianças, apesar dos diversos programas governamentais que têm sido implementados para o controle dessas doenças ao longo dos anos (Telles, Cardozo, \& Souza, 2014).

Nos países subdesenvolvidos, a baixa eficácia de tais iniciativas vincula-se ao aporte financeiro insuficiente para a adoção de medidas de saneamento básico e do tratamento medicamentoso. Concorre para o insucesso desses programas a falta de envolvimento e participação da comunidade por desconhecimento dos problemas que esses parasitos podem causar à saúde das pessoas (Sete, 2015).

Considerando que todos os indivíduos são sujeitos a contrair as enteroparasitoses, mesmo realizando todas as precauções necessárias, como relatado por Andrade et al. (2010), o presente estudo propôs analisar amostras fecais de estudantes de uma escola pública do município de São Mateus, Espírito Santo, Brasil e estabelecer uma associação das parasitoses 
com as condições sanitárias e de higiene da população em estudo, por meio da aplicação de um questionário socioeconômico.

\section{Metodologia}

Optou-se por realizar uma pesquisa de natureza descritiva, privilegiando uma abordagem interpretativa e descrevendo os resultados encontrados dos exames realizados nos alunos envolvidos. Quanto ao embasamento teórico, foi desenvolvido com base na pesquisa bibliográfica, ou de fonte secundária, correspondente à bibliografia já publicada, de forma escrita e/ou oral (jornais, livros, monografias, teses, rádio, televisão, filmes...). Teve como finalidade deixar o pesquisador ciente de tudo que foi abordado sobre o assunto pesquisado (Lakatos, \& Marconi, 2010).

\subsection{REGIÃO ESTUDADA}

A pesquisa foi realizada numa instituição de ensino situada na cidade de São Mateus, estado do Espírito Santo, Brasil.

O município é uma microrregião que está localizada no Nordeste do Espírito Santo, com uma distância de $220 \mathrm{~km}$ da capital Vitória. Limita-se ao Norte com os municípios de Conceição da Barra, Boa Esperança e Pinheiros, ao Sul com os municípios de Linhares, Jaguaré, Vila Valério e São Gabriel da Palha, a Leste com o Oceano Atlântico e ao Oeste com os municípios de Boa Esperança e Nova Venécia (IBGE, 2019).

Segundo o Instituto Brasileiro de Geografias e Estatística apresenta 62,2\% de domicílios com esgotamento sanitário adequado, $70 \%$ de domicílios urbanos em vias públicas com arborização e 17,2\% de domicílios urbanos em vias públicas com urbanização adequada (presença de bueiro, calçada, pavimentação e meio-fio). Quando comparado com os outros municípios do estado, fica nas posições 32 de 78, 30 de 78 e 48 de 78, respectivamente (IBGE, 2019).

A taxa de mortalidade infantil média na cidade é de 13.08 para 1.000 nascidos vivos. As internações devido a diarreias são de 1 para cada 1.000 habitantes. Comparado com todos os municípios do estado, fica nas posições 27 de 78 e 30 de 78, respectivamente (IBGE, 2019).

\subsection{INSTITUIÇÃO DE ENSINO OBJETO DE PESQUISA}

Localizada no município de São Mateus, em bairro distante da região central da cidade, atualmente atende cerca de 540 alunos. No turno matutino a escola trabalha com a faixa etária de 10 a 17 anos e no turno vespertino os alunos têm entre 6 e 15 anos de idade. A instituição oferece ensino do $1^{\circ}$ ao $9^{\circ}$ ano do Ensino Fundamental.

A escola possui um amplo espaço para recreação e possui dois pátios: o interno que é feito de concreto e o externo coberto com areia. A alimentação distribuída para a merenda dos estudantes é feita com acompanhamento de nutricionista e é preparada de acordo com as exigências sanitárias. A água consumida pelos alunos é tratada na estação de água e esgoto do município, sendo depositada em caixas d'água e direcionada aos bebedouros (Prefeitura de São Mateus, 2020).

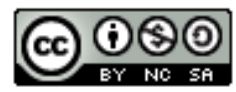



$1-13$.

\subsection{Coleta de Dados e Amostras Biológicas}

Após orientação aos pais e/ou responsáveis pelos 52 estudantes, do $5^{\circ}$ ao $9^{\circ}$ ano, com faixa etária de 11 a 17 anos de idade, de ambos os sexo, sobre a coleta, armazenamento e conservação das fezes (Figura 1) foi entregue o termo de Consentimento Livre e Esclarecido (TCLE) e agendada uma data para que as amostras de fezes fossem recolhidas e transportadas ao Laboratório de Parasitologia e Hematologia Clínica do Centro Universitário Norte do Espírito Santo, da Universidade Federal do Espírito Santo (CEUNES/UFES). Somente aqueles que tiveram o TCLE assinado pelos pais e/ou responsáveis pelo aluno participaram do estudo. Ressalta-se que o projeto tem aprovação no Comitê de Ética em Pesquisa (CAAE: 32929213.2.0000.5063) e está vinculado ao projeto Parasitologia em São Mateus, ES.

Figura 1. Reunião com pais e responsáveis pelos alunos. Fonte: Martins, \& Pivetta. Arquivo Pessoal (2019).

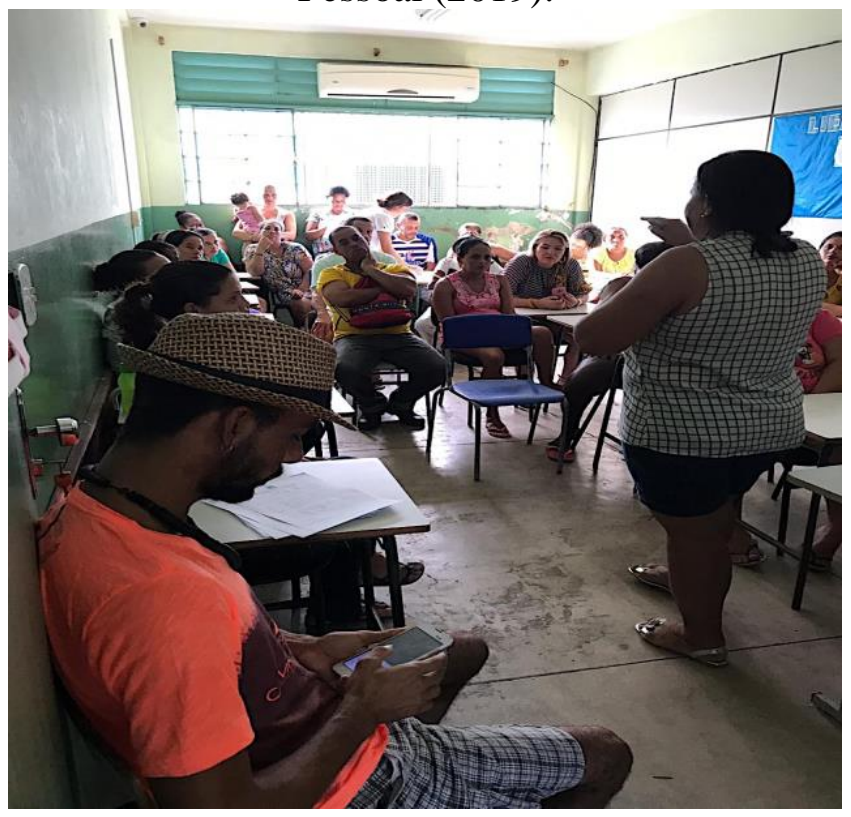

Aplicou-se um questionário para o estudo das condições higiênicas e de moradia da população (Quadro 1) aos alunos. Os resultados foram tabulados, utilizando-se a ferramenta Microsoft Excel®, versão 2016.

I. Identificação
Número do participante:
1- Nome do participante:
2- Endereço da residência:
3- Data da entrevista:
II. Dados pessoais
5- Data de nascimento:
6- Idade (anos):
7- Sexo ( ) masculino ( ) feminino $(\quad$ ) outros
III. Hábitos de higiene
Esta criança costuma:

\section{@ $\odot \Theta \odot$}



$1-13$.

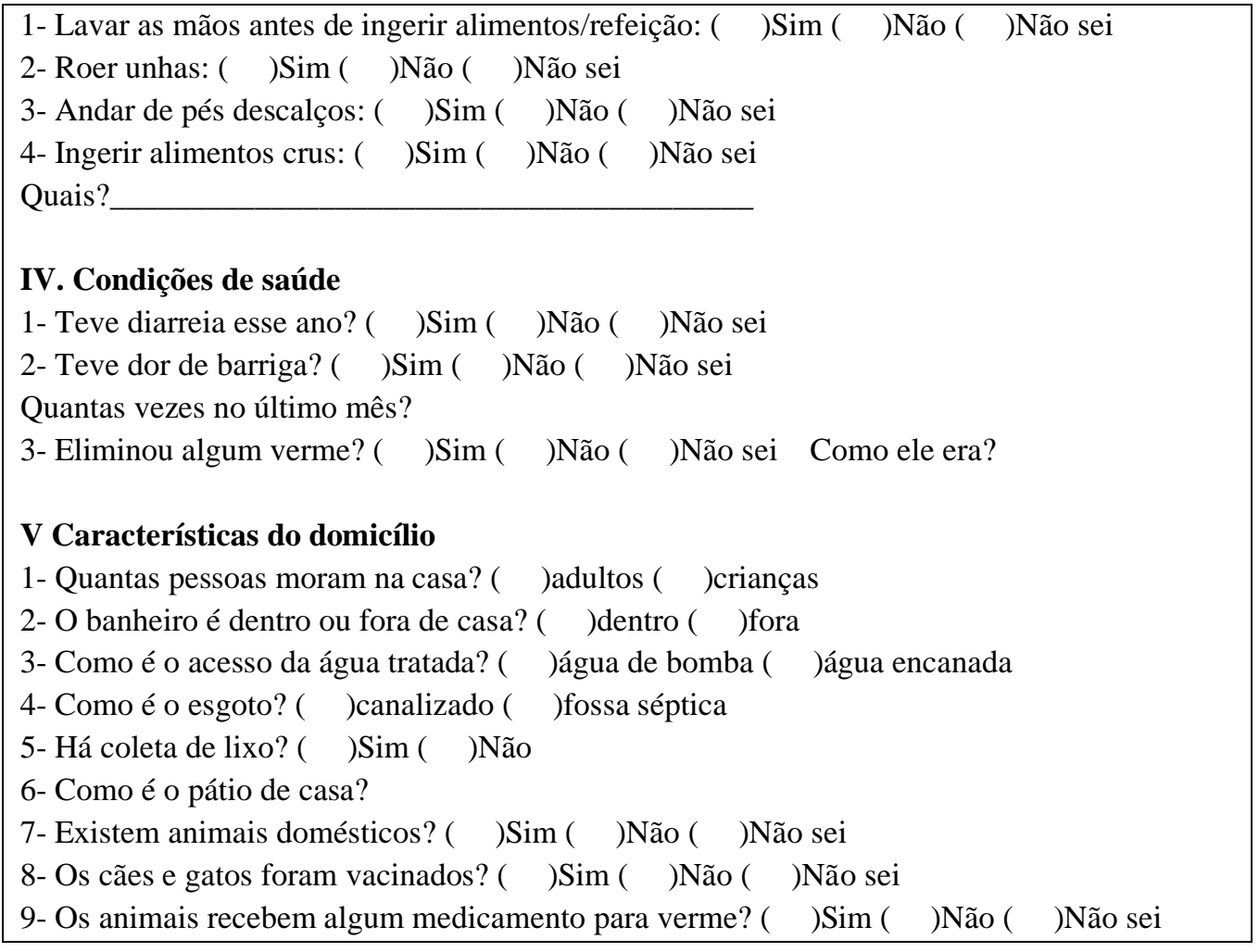

\subsection{Preparo da AMOSTRA E MiCroscopia de LuZ}

As amostras de fezes foram analisadas pelo método de Hoffman, Pons e Janer, também conhecido como método de Lutz, que permite o encontro de ovos e larvas de helmintos, de cistos de protozoários e de alguns oocistos de protozoários que apresentam tamanho maior. Por ser de execução simples e permitir a pesquisa de vários parasitos intestinais do homem é muito utilizado na rotina laboratorial (Neves et al., 2016).

A microscopia das fezes é uma etapa de extrema importância no EPF, onde o profissional deve passar por um treinamento intensivo, tendo conhecimento não só da morfologia das formas parasitárias, mas também do ciclo dos parasitos, com ênfase nas formas parasitárias que são eliminadas com as fezes. Para que seja realizada uma microscopia se deve utilizar equipamento adequado e em perfeito estado. $\mathrm{O}$ microscopista deve saber regular corretamente o equipamento, obtendo deste todos os recursos que possam auxiliá-lo na identificação das formas parasitárias. Vale lembrar que um número excessivo de exames por dia interfere na qualidade do EPF (Neves et al., 2016).

\subsection{Atividades Recreativas e Palestras}

Após as análises e a entrega dos resultados foram realizadas, com os estudantes atividades recreativas - jogos, brincadeiras e teatros - de maneira que estas pudessem entender a importância de prevenção das doenças. Com os pais foram realizadas palestras informando sobre os cuidados para evitar a contaminação. 


\section{Resultados e Discussão}

Este estudo refere-se ao número de estudantes infectados por diferentes parasitos intestinais, residentes em um bairro distante da região central, na cidade de São Mateus.

Previam-se 52 amostras para análise, haja vista que foram realizadas reuniões com os pais explicando o objetivo da pesquisa, os quais mostraram-se interessados. No entanto, mesmo com inúmeras tentativas na Escola, diversos diálogos com a Pedagoga e tentativas de sensibilizar aos alunos, obteve-se um número de adesão ao projeto de apenas 22 amostras (42,31\%), que de certa forma vem de encontro ao observado por Kunz et al. (2008), em seus estudos realizados em Florianópolis, Santa Catarina, os quais afirmam que a baixa adesão de crianças à etapa de coleta e entrega de amostras de fezes foi inesperada face à motivação demonstrada nas demais atividades, porém a dependência do auxílio dos pais para a coleta das amostras pode ter dificultado a tarefa.

Outros estudos, como os de Barreto (2006), realizado na cidade de Guaçuí, Espírito Santo e Ribeiro et al. (2009), na cidade de Cascavel, no Paraná, também indicaram baixa ou média adesão, apesar dos excelentes resultados obtidos.

Com relação a prevalência de enteroparasitoses em São Mateus, em estudantes com idade escolar entre 11 e 15 anos, observou-se uma taxa de infecção de 36,36\% e o percentual de estudantes infectados foi maior quando comparado com outras regiões.

Rech et al. (2016) analisaram a frequência de enteroparasitos e condições socioeconômicas de escolares da cidade de São Marcos, RS e obtiveram um baixo percentual de amostras positivas (5,79\%). Baptista et al. (2006) evidenciaram que $11,54 \%$ das amostras foram positivas para ao menos um parasito, nos estudos no município de Paraíba do Sul, RJ e Cavagnolli, et al. (2015) avaliaram amostras de escolares de Flores da Cunha, RS, com uma taxa de positividade de $10,0 \%$.

Por outro lado, outros estudos, como os realizados por Seixas, et al. (2011) em uma área periurbana de Salvador, Bahia, indicaram uma prevalência de $94 \%$ de infecção por enteroparasitos, sendo a faixa etária mais atingida a de 6 a 9 anos e os parasitos e comensais mais frequentes E. coli (43,5\%), A. lumbricoides (25\%), Endolimax nana (22\%) e E. histolytica/E. dispar (21,5\%).

Em São Mateus, das 22 amostras analisadas, observou-se que os parasitos mais frequentes foram E. histolytica/díspar e T. trichura e o comensal E. coli (Tabela 1). Lodo, et al. (2010) relatam que a amebíase é a protozoose mais comumente encontrada, embora seja frequentemente assintomática.

Tabela 1. Distribuição dos parasitos intestinais de acordo com o sexo.

\begin{tabular}{lcccc}
\hline Parasitos intestinais & $\begin{array}{c}\text { Sexo } \\
\text { Masculino }\end{array}$ & Sexo feminino & Total & $\%$ \\
\hline Ausência de parasitos & 05 & 09 & 14 & $64 \%$ \\
Presença de parasitos & 03 & 05 & 08 & $36 \%$ \\
Entamoeba coli & - & 01 & 01 & $12,5 \%$ \\
Entamoeba histolytica/dispar & 03 & 02 & 05 & $62,5 \%$ \\
Trichuris trichiura & - & 02 & 02 & $25 \%$ \\
\hline
\end{tabular}


Citação (APA): Pivetta, H., Martins, J. dos S., \& Souza, M. A. A. de. (2021). Parasitoses intestinais em estudantes de uma escola pública do município de São Mateus, Espírito Santo, Brasil. Brazilian Journal of Production Engineering, 6(9), Edição Especial "Iniciação Científica", $1-13$.

Segundo Santos, \& Merlini (2010) um dos principais motivos que justificam a alta prevalência de enteroparasitos entre as comunidades decorre da elevada contaminação ambiental, o que necessita prementemente de condições básicas de educação e saneamento.

Com relação a faixa etária, as crianças com 11 anos de idade apresentaram-se mais contaminadas por E. histolytica/dispar (Tabela 2) e as outras faixas etárias apresentaram uma distribuição homogênea de contaminação, em consonância ao observado por MartinichenHerrero, \& Boeira (2013) que relataram que a prevalência de enteroparasitoses varia intensamente de acordo com a idade da criança.

Tabela 2. Distribuição dos parasitos intestinais de acordo com o sexo e idade.

\begin{tabular}{|c|c|c|c|}
\hline \multirow[b]{2}{*}{ Idade/gênero } & \multicolumn{3}{|c|}{ Parasitos intestinais } \\
\hline & Entamoeba histolytica/dispar & Entamoeba coli & Trichuris trichiura \\
\hline 11 anos - Masculino & 02 & - & - \\
\hline 11 anos - Feminino & 02 & - & - \\
\hline Total de indivíduos & 04 & - & - \\
\hline 12 anos - Masculino & 01 & - & - \\
\hline 12 anos - Feminino & - & - & - \\
\hline Total de indivíduos & 01 & - & - \\
\hline 13 anos - Masculino & - & - & - \\
\hline 13 anos - Feminino & - & - & 01 \\
\hline Total de indivíduos & - & - & 01 \\
\hline 14 anos - Masculino & - & - & - \\
\hline 14 anos - Feminino & - & 01 & 01 \\
\hline Total de indivíduos & - & 01 & 01 \\
\hline 15 anos - Masculino & - & - & - \\
\hline 15 anos - Feminino & - & - & - \\
\hline Total de indivíduos & 05 & 01 & 02 \\
\hline
\end{tabular}

Ao apurar os resultados das amostras recolhidas evidencia-se que o sexo feminino foi o mais acometido por algum tipo de parasitose intestinal, equivalente a cerca de $62,5 \%$ dos casos, sendo que a faixa etária com maior índice foi a representada pelas crianças de 11 anos de idade.

Para complementação da pesquisa percebeu-se a necessidade de conhecer a realidade socioeconômica das famílias dos participantes da pesquisa. Dessa forma foi aplicado um questionário com perguntas objetivas sobre hábitos alimentares e higiênicos (Quadro 1).

No quesito hábitos de higiene foi possível apurar que a maior parte dos escolares que responderam à pesquisa (84\%) relatam lavar as mãos antes de ingerirem alimentos e $27 \%$ relataram que ingerem alimentos crus (Tabela 3).

Tabela 3. Hábitos higiênicos das estudantes de acordo com o sexo.

\begin{tabular}{|c|c|c|c|c|c|c|c|c|}
\hline & \multicolumn{2}{|c|}{$\begin{array}{c}\text { Lavam as mãos } \\
\text { antes de ingerir } \\
\text { alimentos }\end{array}$} & \multicolumn{2}{|c|}{ Roem unhas } & \multicolumn{2}{|c|}{$\begin{array}{c}\text { Andam com os pés } \\
\text { descalços }\end{array}$} & \multicolumn{2}{|c|}{$\begin{array}{c}\text { Ingerem } \\
\text { alimentos crus }\end{array}$} \\
\hline & Masc & Fem & Masc & Fem & Masc & Fem & Masc & Fem \\
\hline Sim & 16 & 28 & 12 & 16 & 11 & 14 & 05 & 09 \\
\hline Não & 01 & 07 & 05 & 19 & 06 & 21 & 12 & 26 \\
\hline
\end{tabular}

Hábitos higiênicos são de grande importância para evitar a infecção por doenças parasitárias. Segundo Capuano et al. (2000) as doenças transmitidas por alimentos (DTAs) representam 

$1-13$.

um importante problema de saúde pública tanto nos países desenvolvidos como em desenvolvimento, tendo repercussões tanto na saúde das populações como no desenvolvimento econômico dos países, pois acarretam incapacidade laboral e custos em tratamentos e hospitalizações.

Costa (2011) relata que lavação das mãos é uma barreira eficaz contra as diversas infestações que acometem o ser humano e uma vez praticada constantemente evitará custo aos familiares quanto à compra de medicamentos e a internação da criança no hospital. No entanto, temos o hábito de não nos preocuparmos com a lavação das mãos dando margem para as infecções.

Ferreira et al. (2013) abordam que as enteroparasitoses, de maneira geral, estão ligadas às medidas de higiene, quer seja ambiental ou pessoal. Ambas, em conjunto, interferem diretamente no risco de se ter uma contaminação ou reinfecção por esses agentes etiológicos disponíveis no ambiente domiciliar ou peridomiciliar.

A maioria das infecções causadas por parasitos costuma ser adquirida por via oral, através da contaminação fecal da água e alimentos, sendo importante o trabalho educativo para o combate e prevenção, uma vez que as parasitoses causam diversas manifestações, como a diarreia, má nutrição e retardo no desenvolvimento físico e mental (Ribeiro et al., 2009).

Por outro lado, a diarreia associada com helmintos permanece pouco abordada, porém a maioria dos helmintos tem sido citada como possíveis causadores de diarreia, especialmente quando a infecção intestinal é intensa (Motta, \& Silva, 2002). Nesse sentido, foram analisados os dados dos estudantes do sexo masculino e feminino sobre as condições de saúde e abordou-se a diarreia no questionário apresentado (Tabela 4).

Tabela 4. Condições de saúde das estudantes de acordo com o sexo.

\begin{tabular}{lllllll}
\hline & \multicolumn{3}{c}{ Teve diarreia } & \multicolumn{2}{c}{ Teve dor de barriga } & \multicolumn{2}{c}{ Eliminou algum verme } \\
\cline { 2 - 7 } & Masc & Fem & Masc & Fem & Masc & Fem \\
Sim & 03 & 08 & 09 & 25 & 01 & 01 \\
Não & 10 & 17 & 06 & 10 & 12 & 23 \\
Não sabem & 04 & 10 & 02 & - & 04 & 11 \\
\hline
\end{tabular}

*Masc: maculino; Fem: feminino

Com relação ao número de moradores que residem com os estudantes notou-se que há uma prevalência em ambos os gêneros e uma média de 4 pessoas que habitam na mesma residência. Houve, também, casos de indivíduos com 12 pessoas por residência, contudo este foi o valor menos expressivo (Tabela 5).

Tabela 5. Número de moradores residentes com os estudantes do estudo, de acordo com o

\begin{tabular}{cccc}
\hline $\begin{array}{c}\text { Quantidade de } \\
\text { estudantes do sexo } \\
\text { masculino }\end{array}$ & $\begin{array}{c}\text { Quantidade de } \\
\text { moradores na } \\
\text { residência }\end{array}$ & $\begin{array}{c}\text { Quantidade de } \\
\text { estudantes do sexo } \\
\text { feminino }\end{array}$ & $\begin{array}{c}\text { Quantidade de } \\
\text { moradores na } \\
\text { residência }\end{array}$ \\
\hline 2 & 3 & 1 & 2 \\
7 & 4 & 5 & 3 \\
3 & 5 & 12 & 4 \\
2 & 6 & 7 & 5 \\
2 & 7 & 7 & 6 \\
1 & 12 & 1 & 7 \\
\hline
\end{tabular}


Citação (APA): Pivetta, H., Martins, J. dos S., \& Souza, M. A. A. de. (2021). Parasitoses intestinais em estudantes de uma escola pública do município de São Mateus, Espírito Santo, Brasil. Brazilian Journal of Production Engineering, 6(9), Edição Especial "Iniciação Científica", $1-13$.

Dos estudantes que responderam ao questionário 92\% informaram que têm banheiro dentro de casa. Os escolares que responderam que fazem o uso de água de poço são $44 \%$ ao passo que os que utilizam água encanada são $56 \%$.

Segundo Ribeiro et al. (2009) a maioria das infecções causadas por parasitos costuma ser adquirida por via oral, através da contaminação fecal da água e alimentos, sendo importante o trabalho educativo para o combate e prevenção das patologias (Ribeiro et al., 2009).

Quanto a rede de esgoto na residência, 50\% informaram ter esgoto canalizado, $44 \%$ em forma de fossa séptica e $6 \%$ dos pesquisados não souberam responder. Já com relação a coleta de lixo, $92 \%$ responderam ter coleta de lixo na residência.

Estudos referenciam a importância das medidas de saneamento básico para saúde da criança que quando exposta a condições precárias de esgoto, coleta de lixo e tratamento de água ficam mais vulneráveis aos efeitos deletérios do ambiente, como a contaminação por parasitos (Soares, 2016). Busato et al. (2014) relatam que ambientes que não possuam eliminação adequada de dejetos têm maior probabilidade de contaminação da população, especialmente as crianças, uma vez que a via fecal-oral é o principal meio de infecção pelos parasitos.

Sobre o pátio da casa dos estudantes, $40 \%$ informaram ser de terra batida, outros $40 \%$ informaram ser calçado e $20 \%$ não apresentam pátio em sua residência e isso tem relação direta aos mecanismos de transmissão de formas parasitárias eliminadas por animais de estimação, conforme relatado por Neves et al. (2016).

As parasitoses gastrintestinais causadas por helmintos e protozoários estão entre as enfermidades mais comuns em cães e gatos, podendo ser especialmente graves em animais jovens ou imunocomprometidos. Comumente afetam o equilíbrio orgânico dos animais de estimação, sendo isto particularmente preocupante devido ao íntimo contato destes com o homem (Araújo Do Ó, 2010). No presente estudo 67\% dos estudantes responderam que possuem animais domésticos. Foram respondidas perguntas sobre a vacinação destes animais e $52 \%$ dos estudantes apontaram que os animais são vacinados, $11 \%$ que não são vacinados e $37 \%$ que não souberam responder.

Os animais de estimação, como cães, trazem benefícios psicológicos, fisiológicos e sociais aos seres humanos e, em muitos casos, são considerados como membros da família. Entretanto, apenas um terço dos proprietários tem consciência de que o estreito convívio com o homem aumenta a probabilidade de transmissão de zoonoses parasitárias (Araújo Do Ó, 2010). Segundo estudos realizados por Faleiros et al. (2004) houve uma estreita relação entre o convívio com animais e o encontro de parasitos intestinais.

Mesmo que tal associação possa tratar-se de variável de confusão, uma vez que animais de estimação não representam reservatórios naturais de enteroparasitos, vale lembrar que estes animais têm maior contato com o solo e assim poderiam apresentar formas infectantes de parasitos aderidos aos pelos, favorecendo, desta forma, a transmissão mecânica. No entanto, esta modalidade de transmissão necessita de maior comprovação (Faleiros et al., 2004).

\section{@ $\odot \Theta \odot$}


Citação (APA): Pivetta, H., Martins, J. dos S., \& Souza, M. A. A. de. (2021). Parasitoses intestinais em estudantes de uma escola pública do município de São Mateus, Espírito Santo, Brasil. Brazilian Journal of Production Engineering, 6(9), Edição Especial "Iniciação Científica", $1-13$.

Por fim, é inquestionável a necessidade de mais trabalhos de conscientização nas escolas e, também, entre a população em geral, de modo que ocorra uma expansão do conhecimento acerca das parasitoses intestinais garantindo, assim, uma melhor qualidade de vida da população.

\section{CONCLUSÃo}

Os resultados obtidos podem contribuir para o desenvolvimento de novos estudos sobre a prevalência de parasitoses na população de São Mateus.

Esta pesquisa é relevante para a sociedade podendo indicar a necessidade de alterações e melhorias nos serviços públicos ofertados pelo governo à população. Além disso, pode ajudar a própria população a se conscientizar em relação à existência de enteroparasitoses e o que pode ser feito para prevenir essas doenças.

É fundamental o desenvolvimento de estudos epidemiológicos que estimem a prevalência de parasitoses, buscando avaliar um maior número de sujeitos e desenvolver ações de prevenção junto à comunidade e avaliar o impacto dessas ações, através da realização de novos exames parasitológicos.

Como ainda existem casos de parasitoses, esse estudo deve refletir na melhoria de condições da saúde e maior conscientização da importância da educação sanitária. Traçar perfis como esses podem ajudar na rastreabilidade de locais onde os parasitos são endêmicos e a partir disso desenvolver soluções para minimizar as contaminações.

\section{REFERÊNCIAS BIBLIOGRÁFICAS}

Andrade, E. C., Leite, I. C. G., Rodrigues, V. De O., \& Cesca, M. G. (2010). Parasitoses intestinais: uma revisão sobre seus aspectos sociais, epidemiológicos, clínicos e terapêuticos. Revista APS, 13(2), 231-240.

Araújo, Do Ó. S. (2010). Enteroparasitoses de animais domésticos, com capacidade zoonótica ao homem: um estudo bibliográfico. Trabalho de conclusão de curso (Bacharelado em Farmácia), Universidade Estadual da Paraíba, Campina Grande, 49f.

Baptista, S. C., Breguez, J. M. M., Baptista, M. C. P., Silva, G. M. S., \& Pinheiro, R. O. (2006). Análise da incidência de parasitoses intestinais no município de Paraíba do Sul, RJ. RBAC, 38(4), 271-273.

Barreto, J. G. (2006). Detecção da incidência de enteroparasitos nas crianças carentes da cidade de Guaçuí - ES. RBAC, 38(4), 221-223.

Brasil. Departamento de Informática do Sistema Único de Saúde [Internet]. Brasília, DF: DATASUS; 2011. Informações da Saúde. Demográficas e socioeconômicas. Recuperado de http://www2.datasus.gov.br/ DATASUS/index.php?area=0206.

Brasil. Departamento de Informática do Sistema Único de Saúde [Internet]. Brasília, DF: DATASUS; 2018. Informações da Saúde. Demográficas e socioeconômicas. Recuperado de http://www2.datasus.gov.br/ DATASUS/index.php?area=0206.

Braz, A. S., Andrade, C. A. F., Mota, L. M. H., \& Lima C. M. B. L. (2015). Recomendações da sociedade brasileira de reumatologia sobre diagnóstico e tratamento das parasitoses

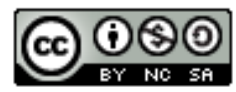


Citação (APA): Pivetta, H., Martins, J. dos S., \& Souza, M. A. A. de. (2021). Parasitoses intestinais em estudantes de uma escola pública do município de São Mateus, Espírito Santo, Brasil. Brazilian Journal of Production Engineering, 6(9), Edição Especial "Iniciação Científica", $1-13$.

intestinais em pacientes com doenças reumáticas autoimunes. Revista Brasileira de Reumatologia, 55(4), 368-380.

Busato, M. A., Antoniolli, M. A., Teo, C. R. P. A., Ferraz, L., Poli, G., \& Tonini, P. (2014). Relação de parasitoses intestinais com as condições de saneamento básico. Ciência, cuidado e saúde, 13(2), 357-363.

Capuano, D. M., Lazzarini, M. P. T., Giacometti Júnior, E., \& Takayanagui, O. M. (2008). Enteroparasitoses em manipuladores de alimentos do município de Ribeirão Preto - SP, Brasil. Revista Brasileira de Epidemiologia, 11(4), 687-689.

Cavagnolli, N. I., Camello, J. T., Tesser, S.; Poeta, J., \& Rodrigues, A. D. (2015). Prevalência de enteroparasitoses e análise socioeconômica de escolares em Flores da Cunha - RS. Revista de Patologia Tropical, 44(3), 312-322.

Cimerman, B., \& Cimerman, S. (2001). Parasitologia humana e seus fundamentos gerais 2.ed., São Paulo: Atheneu, 402p.

Costa, F. B. (2011). Higiene das mãos e na alimentação infantil: a atuação do enfermeiro na Atenção Básica. Trabalho de conclusão de curso (Especialização em Atenção Básica em Saúde da Família), Universidade Federal de Minas Gerais, Governador Valadares, Minas Gerais, $20 \mathrm{f}$.

Damázio, S. M., Soares, A. R., \& Souza, M. A. A. (2016). Perfil parasitológico de escolares da localidade de Santa Maria, zona rural do município de São Mateus/ES, Brasil. Revista APS, 19(2), 261-267.

Faleiros, J. M. M., Gallo, G., Silva, M. M. K., Raful, R., Nasorri, A. R., Pipino, L. F. R., Junqueira, R. B., \& Pinto, P. L. S. (2004). Ocorrência de enteroparasitoses em alunos da escola pública de ensino fundamental do município de Catanduva (São Paulo, Brasil). Revista do Instituto Adolfo Lutz, 63(2) 243-247.

Ferraz, R. R. N., Barnabé, A. S., Porcy, C., D’eça Júnior, A., Feitosa, T., \& Figueiredo, P. M. (2014). Parasitoses intestinais e baixos índices de Gini em Macapá (AP) e Timon (MA), Brasil. Cadernos Saúde Coletiva, 22(2), 173-176.

Ferreira, V. S., Lima, A. G. D., Pessoa, C. S., Paz, F. S. S., \& Jesus, J. (2013). Estudo comparativo das enteroparasitoses ocorrentes em duas áreas de Barreiras, Bahia. Natureza on line, 11(2), 90-95.

IBGE. Instituto Brasileiro de Geografia e Estatística. Brasil em síntese: São Mateus. 2019. Recuperado de https://cidades.ibge.gov.br/brasil/es/sao-mateus/panorama.

Kunz, J. M. O., Vieira, A. S., Varvakis, T., Gomes, G. A., Rossetto, A. L., Bernardini, O. J., Almeida, M. S. S., \& Ishida, M. M. I. (2008). Parasitas intestinais em crianças de escola municipal de Florianópolis, SC - Educação ambiental e em saúde. Biotemas, 21(4), 157-162.

Lakatos, E. M, \& Marconi, M. A. (2010). Fundamentos da Metodologia científica, 7.ed., São Paulo: Atlas, 297p.

Lodo, M., Oliveira, C. G. B., Fonseca, A. L. A., Caputto, L. Z., Packer, M. L. T., Valenti, V. E., \& Fonseca, F. L. A. (2010). Prevalência de enteroparasitas em município do interior paulista. Journal of Human Growth and Development, 20(3), 769-777.

Ludwig, K. M., \& Conte, A. O. C. (2017). Enteroparasitoses em crianças de uma creche na cidade de Assis/SP - antes e depois de campanhas educativas. Saúde, 43(2), 265-345.

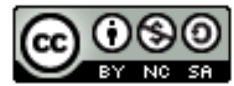


Citação (APA): Pivetta, H., Martins, J. dos S., \& Souza, M. A. A. de. (2021). Parasitoses intestinais em estudantes de uma escola pública do município de São Mateus, Espírito Santo, Brasil. Brazilian Journal of Production Engineering, 6(9), Edição Especial "Iniciação Científica", $1-13$.

Martinichen-Herrero, J. C., \& Boeira, V. L. (2013). Frequência de comensais e parasitas intestinais em escolares da Rede Pública Municipal de Cascavel, PR. RBAC, 45(1-4), 49-52.

Motta, M. E. F. A., \& Silva, G. A. P. (2002). Diarreia por parasitas. Revista Brasileira de Saúde Materno Infantil, 2(2), 117-127.

Neves, D. P., Melo, A. L., Linardi, P. M., \& Vitor, R. W. A. (2016). Parasitologia humana, 13ed., São Paulo: Atheneu, 616p.

Prefeitura de São Mateus. Unidades Escolares. 2020. Recuperado de http://www.saomateus.es.gov.br/acesse/unidades-escolares-emefs.

Rech, S. C., Cavagnolli, N. I., Dalla Santa Spada, P. K., \& Rodrigues, A. D. (2016). Frequência de enteroparasitas e condições socioeconômicas de escolares da cidade de São Marcos-RS. Semina: Ciências Biológicas e da Saúde, 37(1), 25-32.

Ribeiro, M. S., Gonçalves, R. R., Morais, F. G., \& Boeira, V. L. (2009). Controle de parasitoses intestinais através da educação em saúde em crianças do ensino fundamental de Cascavel - PR. Biotemas, 21(4), 157-162.

Santos, P. H. S., Barros, R. C. S., Gomes, K. V. G., Nery, A. G., \& Casotti, C. A. (2017). Prevalência de parasitoses intestinais e fatores associados em idosos. Revista Brasileira de Geriatria e Gerontologia, 20(2), 244-254.

Santos, S. A., \& Merlini, L. S. (2010). Prevalência de enteroparasitoses na população do município de Maria Helena, Paraná. Ciência \& Saúde Coletiva, 15(3), 899-905.

Seixas, M. T. L., Souza, J. N., Souza, R. P., Teixeira, M. C. A., \& Soares, N. C. (2011). Avaliação da frequência de parasitos intestinais e do estado nutricional em escolares de uma área periurbana de Salvador, Bahia, Brasil. Revista de Patologia Tropical, 40(4), 304-314.

Sete, D G. (2015). Proposta de intervenção para redução das enteroparasitoses em instituição de educação em Belo Oriente, Minas Gerais. 2015. Trabalho de conclusão de curso (Especialização em Saúde da Família), Universidade Federal de Minas de Gerais, Belo Horizonte, Minas Gerais, 53f.

Soares, T. V. C. (2016). Análise de parasitas intestinais em amostras fecais infantis e comparação entre métodos parasitológicos. 2016. Trabalho de conclusão de curso (Bacharelado em Farmácia), Universidade de Brasília, Ceilândia, Distrito Federal, 49f.

Tavares, A. D., Frazão, D. M., Britto, H. D. M. S., Cavalcanti, M. O., Colares, M. C. R., \& Pereira, T. G. R. Prevalência de parasitose em uma escola pública frequentada por crianças de baixo nível sócio-econômico. In: Congresso Brasileiro de Extensão Universitária UFPB, 2001, Campina Grande, Paraíba. Anais do I Congresso Brasileiro de Extensão Universitária UFPB, Campina Grande, PB, 2001, (CD-ROOM).

Telles, V. G. A.; Cardozo, R. O.; \& Souza, M. A. A. (2014). Estudo epidemiológico sobre enteroparasitoses no município de São Mateus, Espírito Santo, Brasil. Scientia Vitae, 2(6), 25 32 . 